Delft University of Technology

\title{
The heterogeneous to homogeneous transition for slurry flow in pipes
}

\author{
Miedema, Sape
}

DOI

10.1016/j.oceaneng.2016.07.031

Publication date

2016

Document Version

Accepted author manuscript

Published in

Ocean Engineering

\section{Citation (APA)}

Miedema, S. (2016). The heterogeneous to homogeneous transition for slurry flow in pipes. Ocean

Engineering, 123, 422-431. https://doi.org/10.1016/j.oceaneng.2016.07.031

\section{Important note}

To cite this publication, please use the final published version (if applicable).

Please check the document version above.

Other than for strictly personal use, it is not permitted to download, forward or distribute the text or part of it, without the consent of the author(s) and/or copyright holder(s), unless the work is under an open content license such as Creative Commons.

\section{Takedown policy}

Please contact us and provide details if you believe this document breaches copyrights.

We will remove access to the work immediately and investigate your claim. 


\title{
The Heterogeneous to Homogeneous Transition for Slurry Flow in Pipes.
}

\author{
Sape A. Miedema \\ Delft University of Technology
}

\begin{abstract}
Models for modeling slurry flow present difficulties on long lines with large pipe diameters and with broad graded sands or gravels. In order to get more insight into the slurry flow process, the Delft Head Loss \& Limit Deposit Velocity Framework has been developed that integrates the 5 main flow regimes of slurry transport: fixed or stationary bed transport, sliding bed transport, heterogeneous transport, homogeneous transport and sliding flow transport. Additional models for; the limit deposit velocity, the holdup function, the bed height, the concentration distribution and graded sands and gravels, complement the Framework. The Framework is based on constant spatial volumetric concentration curves for uniform sands and gravels. The models for the flow regimes and the limit deposit velocity are based on energy considerations.
\end{abstract}

At line speeds near the transition of the heterogeneous and homogeneous flow regimes however, there is no sharp transition between the two flow regimes for medium sized particles. The limits of medium sized particles depend on the solid and liquid properties and on the pipe diameter. It is often observed that the hydraulic gradient lies in between the Equivalent Liquid Model (ELM) and the pure liquid Darcy Weisbach model at higher line speeds, resulting in the conclusion that at higher line speeds the pure liquid hydraulic gradient is approached. Based on energy considerations however, it is shown that the heterogeneous hydraulic gradient collapses due to turbulent near wall lift neutralizing the particle submerged weight and collisions with the pipe wall, while at higher line speeds the turbulent eddies integrate particles, resulting in a hydraulic gradient approaching a reduced ELM (RELM). For medium sized particles in large diameter pipes there is a gap between the moment the heterogeneous hydraulic gradient collapses and the homogeneous hydraulic gradient builds up, resulting in a hydraulic gradient approaching the RELM hydraulic gradient. The model for this transition is described and derived and experimental evidence is given.

\section{KEYWORDS: Slurry Transport, Hydraulic Gradient, Limit Deposit Velocity}

\section{INTRODUCTION}

Although sophisticated 2 and 3 layer models exist for slurry flow (here the flow of sand/gravel water mixtures), the main Dutch and Belgian dredging companies still use modified Durand \& Condolios (1952) and Fuhrboter (1961) models for heterogeneous transport, while the main companies in the USA use a modified Wilson et al. (2006) model, and the coal industry uses the Wasp (1977) model or the SRC (1991) model. The empirical models use one term for the excess head losses resulting from the solids (the solids effect). Most models are based on experiments in small diameter pipes. The use of these models does not give a satisfying result on projects with large diameter pipelines $\left(D_{p}>0.75 \mathrm{~m}\right.$ ), very long pipelines (up to $36 \mathrm{~km}$ ) or broad PSD's (particle size distributions). This is the reason to study all existing models and develop a Framework that agrees with most existing models, but also solves the shortcomings. The result is the DHLLDV (Delft Head Loss \& Limit Deposit Velocity) Framework. The DHLLDV Framework determines the resulting hydraulic gradient curve for spatial volumetric concentrations and uniform sands, consisting of parts of all flow regimes occurring for the pipe and particle diameter in question.

Since the slurry flow process in dredging is a non-stationary process, the PSD and concentration may be time and space dependent, resulting in time varying line speeds, density waves, moving dunes and so on, the modelling should be based on time averaged properties and parameters. In the Framework the flow regime models are based on energy considerations. Each flow regime has its own physical and mathematical model. Once the resulting hydraulic gradient curve and the Limit Deposit Velocity (LDV) are determined, a holdup function is used to construct the transport volumetric concentration curve and the bed height. The latest addition to the Framework is a method to determine the concentration distribution based on the LDV and the Wasp (1963) model. The LDV is defined here as the line speed above which there is no stationary or sliding bed.

For graded sands and gravels the curves are determined for each uniform grain fraction individually, and the resulting curves are then combined by superposition to form the overall hydraulic gradient curve, holdup function and bed height curve. 


\section{THE DHLLDV FRAMEWORK}

The Delft Head Loss \& Limit Deposit Velocity Framework (DHLLDV) is a Framework based on constant spatial volumetric concentration curves and uniform sands or gravels for 5 flow regimes in a Newtonian fluid (Ramsdell \& Miedema (2013) and Miedema \& Ramsdell (2014B)). These 5 regimes are the stationary or fixed bed regime (Miedema \& Matousek (2014)), the sliding bed regime (Miedema \& Ramsdell (2014A) and Miedema (2014B)), the heterogeneous regime (Miedema \& Ramsdell (2013) and Miedema (2014A) and (2015B)), the homogeneous regime (Miedema (2015A)) and the sliding flow regime (Miedema \& Ramsdell (2014B). Crucial for the modelling is the determination of the Limit Deposit Velocity (LDV). The constant delivered volumetric concentration curves are determined based on the LDV ( (Miedema \& Ramsdell, 2015A)) and the holdup function (Miedema, 2015C). The bed height is determined based on the LDV and the holdup function. Curves for graded sands and gravels are determined by superposition of the curves of the fractions of the Particle Size Distribution (PSD). The Limit Deposit Velocity is defined here as the line speed above which there is no stationary bed or sliding bed, Thomas (1962). Below the LDV there may be either a stationary or fixed bed or a sliding bed.

The DHLLDV Framework is based on energy considerations. In each flow regime the main source of energy losses due to the solids is identified.

1. The energy losses in the fixed or stationary flow regime are due to turbulence of the liquid above the bed.

2. The energy losses in the sliding bed regime are due to sliding friction of the solids with the pipe wall.

3. The energy losses in the heterogeneous flow regime are due to potential energy (gravity) and kinetic energy (collisions of the solids with the pipe wall).

4. The energy losses in the homogeneous flow regime are due to turbulence of the liquid carrying the solids. Large eddies are formed, with a size related to the pipe diameter and a circumferential velocity related to the line speed. The large eddies break up into smaller eddies which break up in even smaller eddies until the smallest eddies are formed that will dissipate into heat. So the energy dissipated starts with the rotational energy of the largest eddies, which is proportional to the density of the rotating mass, the mixture density. This philosophy is the basis of the Equivalent Liquid Model (ELM). If the dissipated energy is proportional to the mixture density, then the liquid density can be replaced by the mixture density in the well-known Darcy Weisbach equation. Because of lift, due to the velocity gradient near the pipe wall, directed to the center of the pipe, particles are forced away from the viscous sub layer resulting in an almost particle free viscous sub layer. This effect reduces the Darcy Weisbach friction factor and results in pressure losses lower than the ELM (Miedema, 2015A). Wilson \& Sellgren (2003) also identified this as the near wall lift effect.

5. The energy losses in the sliding flow regime are due to both sliding friction and potential and kinetic energy losses.

\section{HETEROGENEOUS TRANSPORT}

Heterogeneous transport is defined as transport where potential energy and kinetic energy losses dominate the head losses. Miedema \& Ramsdell (2013) derived an equation for the Relative Excess Hydraulic Gradient for heterogeneous transport based on energy considerations. This equation consists of two parts. A first part for the contribution due to potential energy losses and a second part for the kinetic energy losses. The equation is based on uniform sands or gravels, but Miedema (2014A) also derived a modified equation for graded sands and gravels. In its basic form the equation looks like:

$$
E_{r h g}=\frac{i_{m}-i_{1}}{R_{s d} \cdot C_{v s}}=\frac{v_{t} \cdot\left(1-\frac{C_{v s}}{\kappa_{C}}\right)^{\beta}}{v_{l s}}+\left(\frac{v_{s l}}{v_{t}}\right)^{2}=S_{h r}+S_{r s}
$$

The Settling Velocity Hindered Relative, $\mathrm{S}_{\mathrm{hr}}$, is the Hindered Settling Velocity of a particle $\mathrm{v}_{\mathrm{t}} \cdot\left(1-\mathrm{C}_{\mathrm{vs}} / \kappa_{\mathrm{C}}\right)^{\beta}$ divided by the line speed $v_{l s}$. The $S_{h r}$ value gives the contribution of the potential energy losses to the Relative Excess Hydraulic Gradient. The $S_{h r}$ is derived for and can be applied to the heterogeneous regime. The Slip Relative Squared $S_{\mathrm{rs}}$ is the Slip Velocity of a particle $\mathrm{v}_{\mathrm{sl}}$ divided by the Terminal Settling Velocity of a particle $\mathrm{V}_{\mathrm{t}}$ squared and this $S_{\mathrm{rs}}$ value is a good indication of the Relative Excess Hydraulic Gradient due to the solids, since its contribution to the total is $90 \%-100 \%$. The $\mathrm{S}_{\mathrm{rs}}$ value gives the contribution of the kinetic energy losses to the Relative Excess Hydraulic Gradient $\mathrm{E}_{\mathrm{rhg}}$. The $\mathrm{S}_{\mathrm{rs}}$ is derived for and can be applied to the heterogeneous regime. The potential energy term is explicit and all the variables involved are known, so this term can be solved. The kinetic energy term however contains the slip velocity, which is not known. The kinetic energy term has been derived by Miedema \& Ramsdell (2013) based on kinetic energy losses due to collisions or interactions with the 
pipe wall or the viscous sub layer. This means that the slip velocity used in the above equation is not necessarily the average slip velocity, but it is the slip velocity necessary to explain the kinetic energy losses. The average slip velocity of the particles will probably be larger, but of the same magnitude.

The relative excess hydraulic gradient $\mathrm{E}_{\mathrm{rhg}}$ is mathematically the same as the stratification ratio $\mathrm{R}$ as used by Wilson et al. (1992) and later to give an indication of the stratification ratio in the heterogeneous regime. A new name is introduced, because the naming should match the physics. In the stationary regime and the homogeneous regime the value of the stratification ratio $\mathrm{R}$ increases with the line speed at constant spatial volumetric concentration, while in reality it's more or less fixed. A stationary bed has $100 \%$ stratification, while in the homogeneous regime the stratification is supposed to be $0 \%$. But also in the heterogeneous flow regime the $\mathrm{R}$ does not give the amount of stratification. At $100 \%$ stratification in a sliding bed, the value equals the sliding friction coefficient, while at $0 \%$ stratification, the transition of heterogeneous to homogeneous flow above the limit deposit velocity, the $\mathrm{R}$ is not zero. This is the reason why the relative excess hydraulic gradient $\mathrm{E}_{\text {rhg }}$ is introduced, explaining the physics better.

The derivation of the slip velocity equation for uniform sands or gravels is the subject of Miedema (2015B). Giving for the relative excess hydraulic gradient, the $\mathrm{E}_{\mathrm{rhg}}$ parameter:

$$
E_{\text {rhg }}=\frac{v_{t} \cdot\left(1-\frac{C_{v s}}{0.175 \cdot(1+\beta)}\right)^{\beta}}{v_{\text {Is }}}+6.8^{2} \cdot\left(\frac{\delta_{v}}{d}\right)^{2 / 3} \cdot\left(\frac{v_{t}}{11.6 \cdot u_{*}}\right)^{4 / 3} \cdot\left(\frac{R_{s d}}{C_{D}}\right)
$$

With the thickness of the viscous sub layer and the velocity at this thickness (using another definition would just change the constant of 6.8 ):

$$
\delta_{v}=11.6 \cdot \frac{v_{1}}{u_{*}}=11.6 \cdot \frac{v_{1}}{\sqrt{\frac{\lambda_{1}}{8}} \cdot v_{1 s}} \quad \text { and } \quad v_{\delta_{v}}=\frac{u_{*}^{2}}{v_{1}} \cdot \delta_{v}=11.6 \cdot u_{*}
$$

The first term on the right hand side of equation (4) is the contribution due to potential energy losses, and the second term the contribution due to kinetic energy losses. The second term consists of 3 terms. The first term is the ratio of the thickness of the viscous sub layer to the particle diameter. This term stands for the lubrication effect of a reduced concentration in the viscous sub layer and has a maximum value of 1 when particles fit completely in the viscous sub layer. Larger particles result in a reduction of the concentration in the viscous sub layer due to the geometry, without near wall lift. The second term is the angle of attack of particles with the pipe wall. The larger the angle of attack, the higher the kinetic energy losses. The third term gives the proportionality of the kinetic energy losses with the relative submerged density and the particle drag coefficient. The higher the relative submerged density, the higher the kinetic energy losses. The higher the particle drag coefficient, the more particles are influenced by turbulence and the less they have collisions with the pipe wall. So the kinetic energy losses are reversely proportional to the particle drag coefficient. Using a particle shape factor of 0.75 , this can be written as:

$$
E_{r h g}=\frac{v_{t} \cdot\left(1-\frac{C_{v s}}{0.175 \cdot(1+\beta)}\right)^{\beta}}{v_{\text {ls }}}+8.5^{2} \cdot\left(\frac{1}{\lambda_{1}}\right) \cdot\left(\frac{v_{t}}{\sqrt{g \cdot d}}\right)^{10 / 3} \cdot\left(\frac{\left(v_{1} \cdot g\right)^{1 / 3}}{v_{l s}}\right)^{2}
$$

\section{HOMOGENEOUS TRANSPORT}

Homogeneous transport is defined as transport where the concentration distribution is close to being uniform and the head losses behave similar to the Darcy Weisbach head losses, but with some correction. The basis of the homogeneous transport regime model is the equivalent liquid model (ELM). In terms of the relative excess hydraulic gradient, $\mathrm{E}_{\mathrm{rhg}}$, this can be written as: 


$$
E_{r h g}=\frac{i_{m}-i_{1}}{R_{s d} \cdot C_{v s}}=\frac{\lambda_{1} \cdot v_{l s}^{2}}{2 \cdot g \cdot D_{p}}=i_{1}
$$

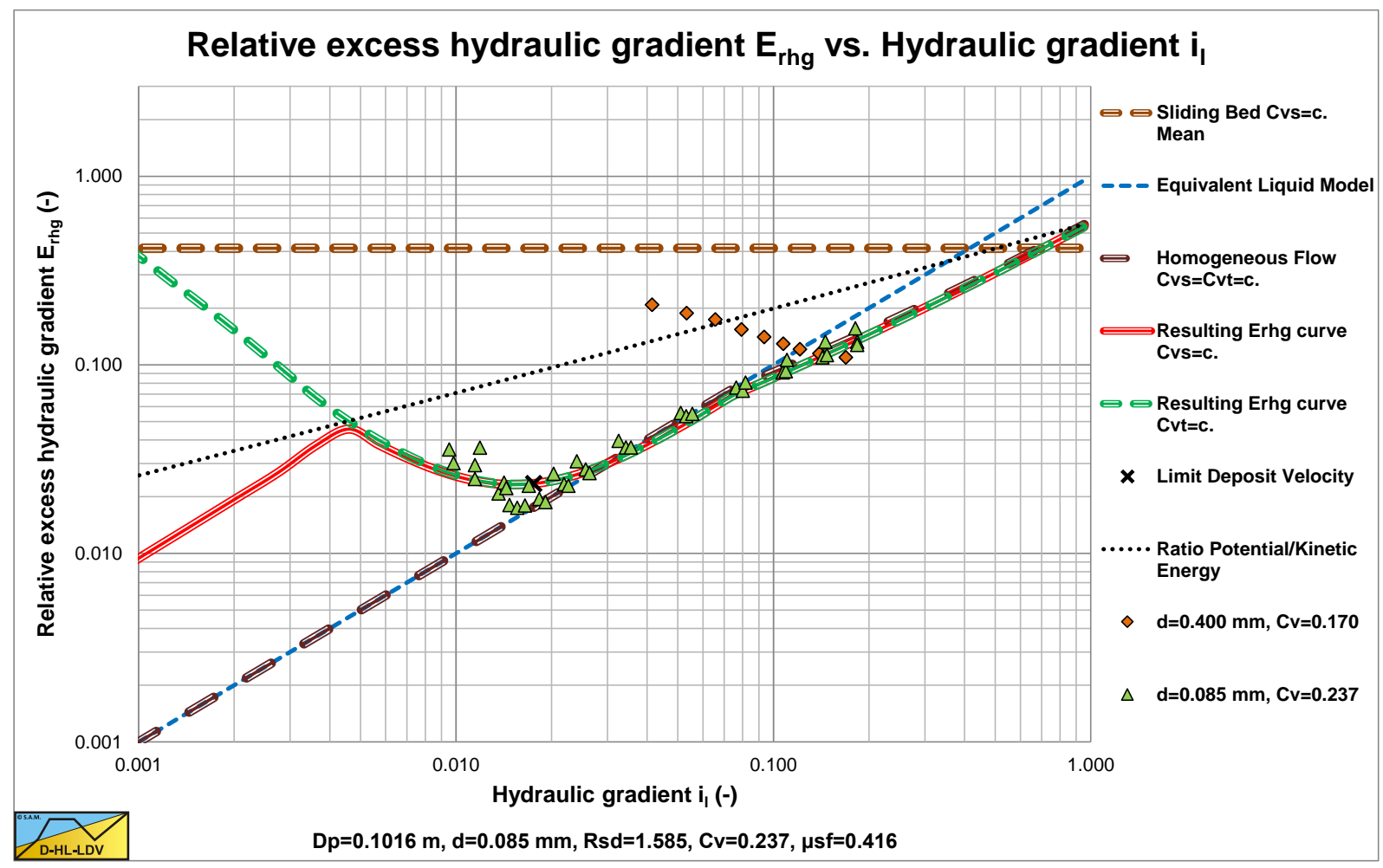

Figure 1: Experimental data of Whitlock et al. (2004).

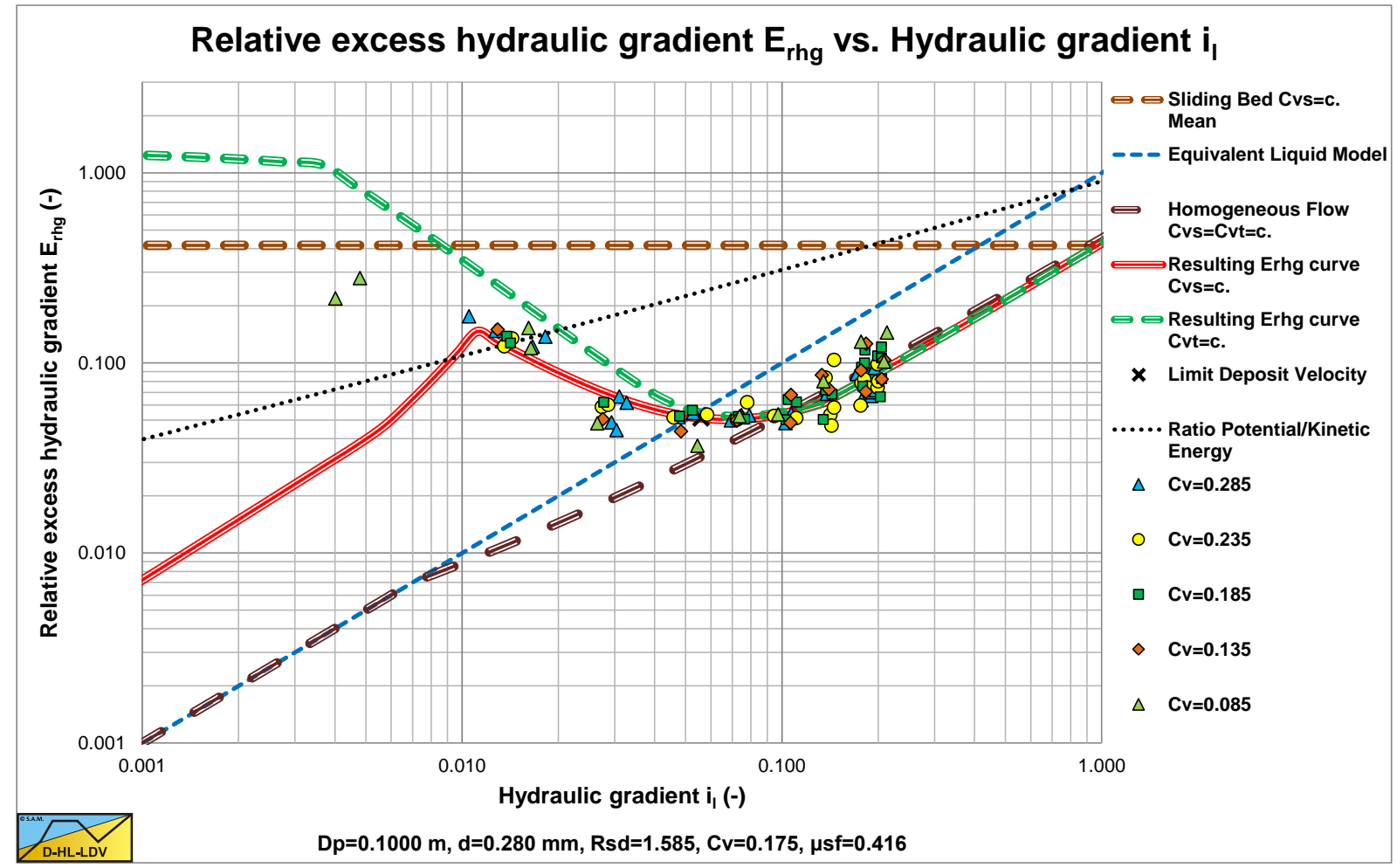

Figure 2: Experiments of Blythe \& Czarnotta (1995).

Talmon (2013) derived an equation to correct the homogeneous equation (the ELM model) for the slurry density, based on the hypothesis that the viscous sub-layer hardly contains solids at very high line speeds in the homogeneous regime. This theory results in a reduction of the resistance compared with the ELM, but the 
resistance is still higher than the resistance of clear water. Talmon (2013) used the Prandl approach for the mixing length, which is a 2D approach for open channel flow with a free surface. The Prandl approach was extended with damping near the wall to take into account the viscous effects near the wall, according to von Driest (Schlichting, 1968). Miedema (2015A) improved the equation for pipe flow and a concentration distribution giving for the relative excess hydraulic gradient $\mathrm{E}_{\mathrm{rhg}}$ :

$$
E_{r h g}=\frac{i_{m}-i_{l}}{R_{s d} \cdot C_{v s}}=i_{l} \cdot \frac{1+R_{s d} \cdot C_{v s}-\left(\frac{A_{C_{v}}}{\kappa} \cdot \ln \left(\frac{\rho_{m}}{\rho_{l}}\right) \cdot \sqrt{\frac{\lambda_{l}}{8}}+1\right)^{2}}{R_{s d} \cdot C_{v s} \cdot\left(\frac{A_{C_{v}}}{\kappa} \cdot \ln \left(\frac{\rho_{m}}{\rho_{l}}\right) \cdot \sqrt{\frac{\lambda_{l}}{8}}+1\right)^{2}}
$$

The resulting equation (6) with $A_{C v}=3$ gives a good average behavior based on the data of Talmon (2011) and Thomas (1976). Since the model is based on a particle free viscous sub-layer and the viscosity of the carrier liquid, it may not give good predictions for very small particles. Very small particles may influence the viscosity and fit completely in the viscous sub layer, especially at low line speeds. It is observed that very small particles behave according to the ELM, if necessary corrected for the viscosity and density. Medium and large particles show the reduction according to equation (6). The transition of the ELM to the reduced ELM appears to depend on the ratio of the thickness of the viscous sub layer to the particle diameter with a maximum of 1 . This ratio is an indication of the concentration reduction in the viscous sub layer, giving:

$$
\begin{aligned}
E_{r h g} & =\frac{i_{m}-i_{l}}{R_{s d} \cdot C_{v s}} \\
& =\left.i_{1} \cdot\right|_{1} \mid \begin{array}{l}
1 \\
\mid
\end{array}
\end{aligned}
$$

Figure 1 shows experimental data of Whitlock et al. (2004) showing that very small particles $(\mathrm{d}=0.085 \mathrm{~mm})$ have a rather sharp transition from the heterogeneous regime to the ELM, while at higher line speeds the reduction due to a lower concentration in the homogeneous regime is mobilized. Larger particles $(\mathrm{d}=0.4 \mathrm{~mm})$ however seem to have some overshoot. Figure 2 shows experiments of Blythe \& Czarnotta (1995). From these experiments it is clear that the relative excess hydraulic gradient crosses the ELM curve, after which it tends to go back to the direction of the homogeneous curve (reduced ELM or RELM) asymptotically.

\section{THE TRANSITION HETEROGENEOUS-HOMOGENEOUS}

In the previous paragraphs the heterogeneous regime and the homogeneous regime are discussed. The question is now, is there a sharp transition between the two flow regimes, or is this more complicated. Figure 3 and Figure 4 show experiments of Clift et al. (1982) in pipes with $0.2032 \mathrm{~m}$ and $0.44 \mathrm{~m}$ diameters. The particle diameter in these experiments was $0.42 \mathrm{~mm}$. The graphs show that the behavior near the ELM curve depends on the pipe diameter. The experiments in the $0.2 \mathrm{~m}$ diameter pipe show a rather smooth transition, while the experiments in the 0.44 diameter pipe show a collapse of the relative excess hydraulic gradient near the intersection point of the two flow regimes. Wilson et al. (1992) explain this phenomena with near wall lift, based on the ratio of the submerged weight of a particle to the lift force. There may, however be other phenomena influencing this behavior.

The classification of (very) small, medium and large particles is not an absolute classification. Small particles are particles that fit or almost fit in the viscous sub-layer. Medium sized particles are particles small enough to follow the turbulent eddies, but to large to fit in the viscous sub-layer. Large particles are particles that do not follow the turbulence. 


\section{THE LIFT RATIO}

When the particle diameter approaches the thickness of the viscous sub-layer at the transition velocity heterogeneous-homogeneous a peculiar phenomenon occurs. On one hand the kinetic losses collapse due to the lift forces available just above the viscous sub-layer, assuming there are no lift forces in the viscous sub-layer, on the other hand the eddies in the turbulent layer are not yet strong enough to integrate the particles. When the line speed increases, the lift forces are strong to counteract the submerged weight and kinetic energy of the particles, but not enough to integrate the particles in the eddy behavior. This results in a sort of delay between the collapse of the kinetic losses and the mobilization of the RELM.

Wilson et al. (2010) introduced the lift force on a particle as:

$$
F_{L}=C_{L} \cdot \frac{1}{2} \cdot \rho_{1} \cdot u_{*}^{2} \cdot \frac{\pi}{4} \cdot d^{2}
$$

And for the submerged weight of the particle (including the shape factor):

$$
\mathbf{F}_{G}=\left(\rho_{\mathrm{s}}-\rho_{1}\right) \cdot \mathbf{g} \cdot \frac{\pi}{6} \cdot \mathbf{d}^{3} \cdot \psi
$$

Giving, assuming a shape factor of 0.75 :

$$
\begin{aligned}
\mathbf{L}_{\mathbf{R}} & =\frac{\mathbf{F}_{\mathrm{L}}}{\mathbf{F}_{\mathrm{G}}}=\frac{\mathbf{C}_{\mathrm{L}} \cdot \frac{1}{2} \cdot \rho_{1} \cdot \mathbf{u}_{*}^{2} \cdot \frac{\pi}{4} \cdot \mathbf{d}^{2}}{\left(\rho_{\mathrm{s}}-\rho_{1}\right) \cdot \mathrm{g} \cdot \frac{\pi}{6} \cdot \mathbf{d}^{3} \cdot \psi}=\mathbf{C}_{\mathrm{L}} \cdot \frac{3}{4} \cdot \frac{\mathbf{u}_{*}^{2}}{\mathbf{R}_{\mathrm{sd}} \cdot \mathrm{g} \cdot \mathbf{d} \cdot \psi} \\
& =\mathbf{C}_{\mathrm{L}} \cdot \frac{3}{32} \cdot \frac{\lambda_{1} \cdot \mathbf{v}_{\mathrm{Is}}^{2}}{\mathbf{R}_{\mathrm{sd}} \cdot \mathrm{g} \cdot \mathbf{d} \cdot \psi}=\mathbf{C}_{\mathrm{L}} \cdot \theta
\end{aligned}
$$

The difference in kinetic energy of a particle with a horizontal and a vertical velocity component (the hindered settling velocity) compared with the kinetic energy of a particle with only the horizontal velocity component is:

$$
E_{K}=\frac{1}{2} \cdot m_{p} \cdot v_{t h}^{2}=\frac{1}{2} \cdot \rho_{s} \cdot \frac{\pi}{6} \cdot d^{3} \cdot \psi \cdot v_{t h}^{2}
$$

The force to reduce the vertical component of the kinetic energy of a particle to zero over a certain distance $\mathrm{x}$ equals:

$$
F_{K}=\frac{1}{2} \cdot m_{p} \cdot \frac{v_{\text {th }}^{2}}{x}=\frac{1}{2} \cdot \rho_{s} \cdot \frac{\pi}{6} \cdot d^{3} \cdot \psi \cdot \frac{v_{\text {th }}^{2}}{x}=\frac{E_{K}}{x}
$$

Which is the kinetic energy divided by the distance $\mathrm{x}$, so it also matches the principle that the work carried out equals the force times the distance is equal to the destroyed kinetic energy. 


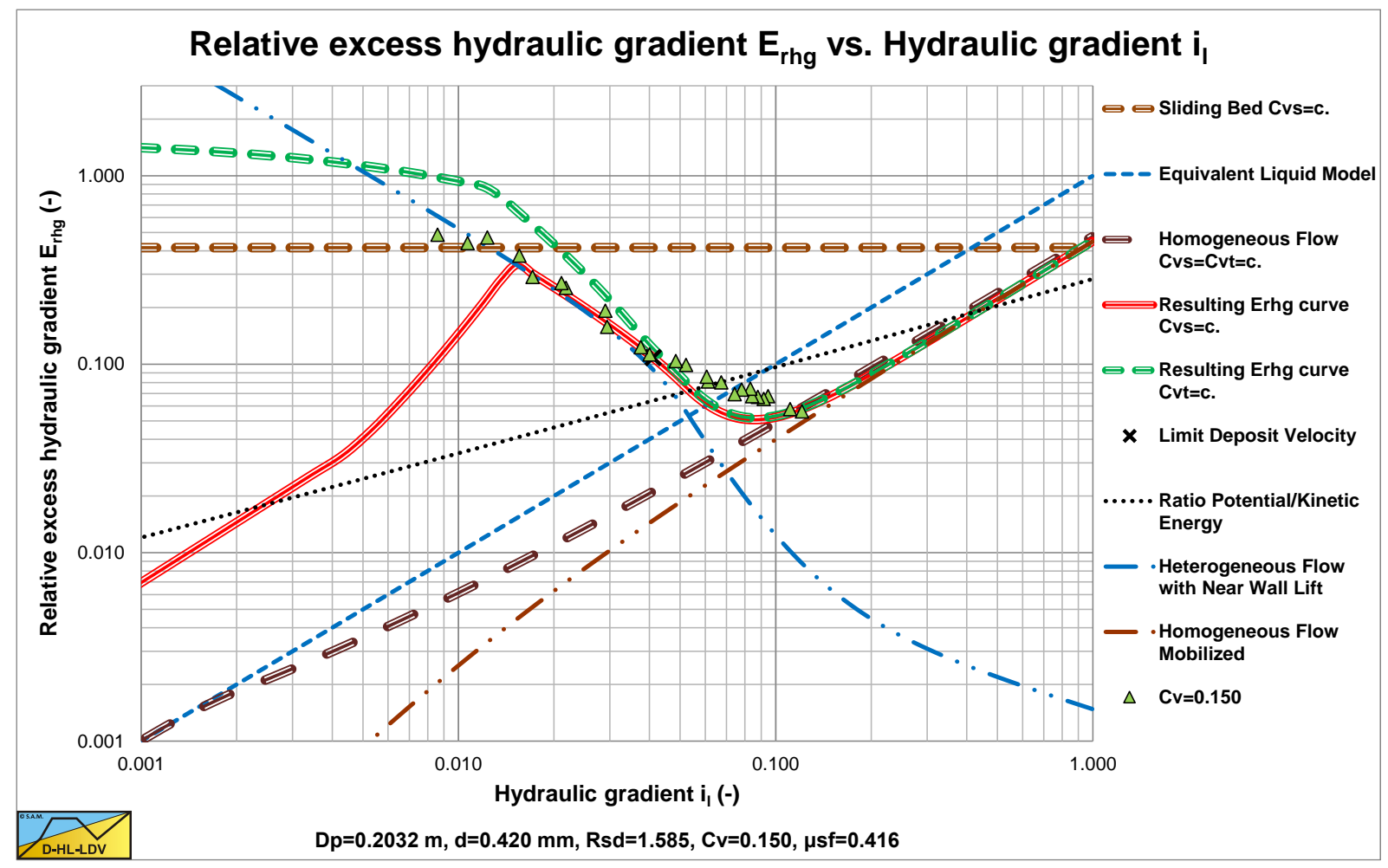

Figure 3: Experiments of Clift et al. (1982) in a $0.2082 \mathrm{~m}$ diameter pipe and a $0.42 \mathrm{~mm}$ particle.

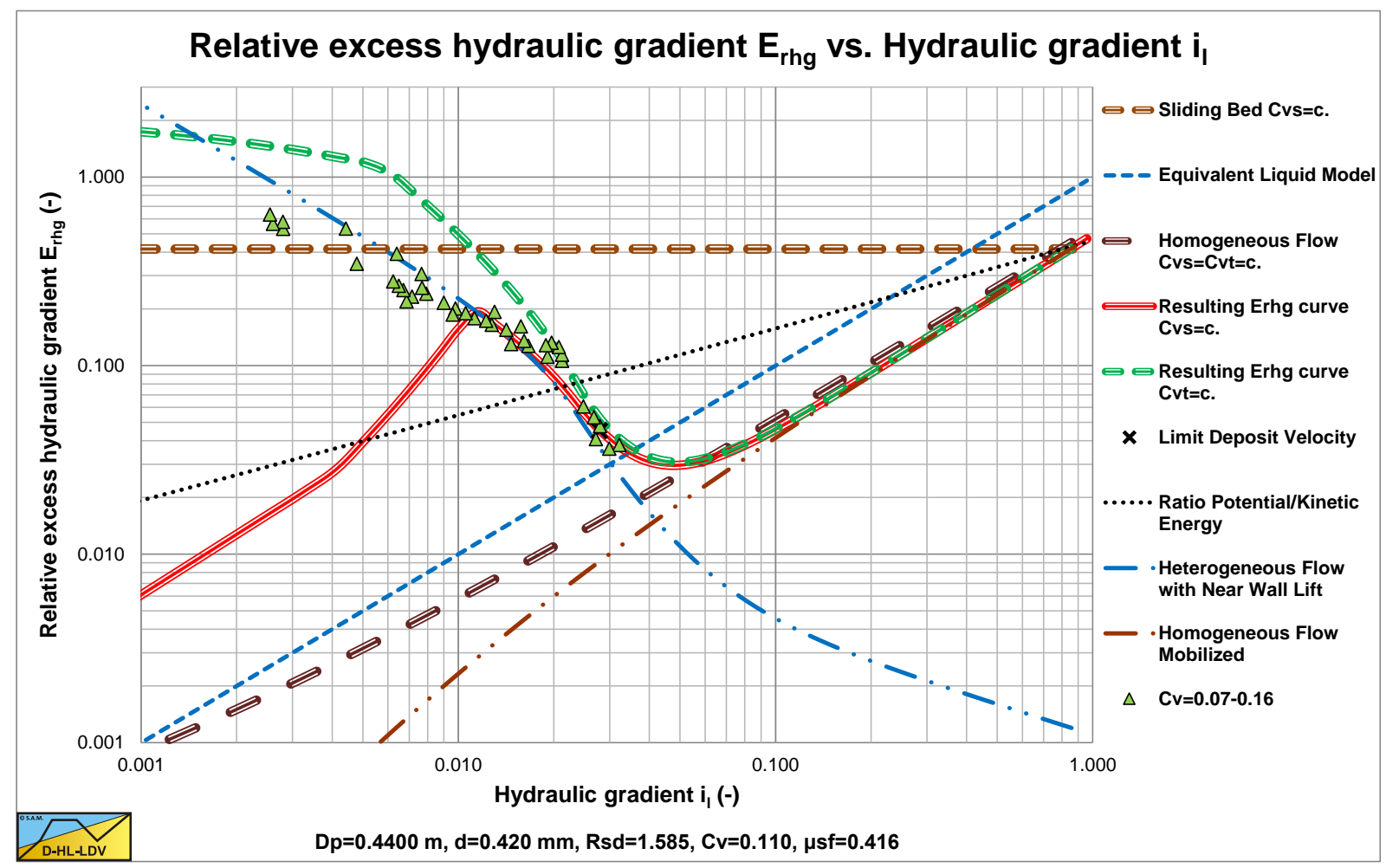

Figure 4: Experiments of Clift at al. (1982) in a $0.4400 \mathrm{~m}$ diameter pipe and a $0.42 \mathrm{~mm}$ particle. 
To prevent a particle from having a collision with the pipe wall, the lift force has to counteract both the submerged weight of the particle and the kinetic energy force, giving for the lift ratio:

$$
\begin{aligned}
& \mathbf{L}_{\mathbf{R}}=\frac{\mathbf{F}_{\mathrm{L}}}{\mathbf{F}_{\mathbf{G}}+\mathbf{F}_{\mathrm{K}}}=\frac{\mathbf{C}_{\mathrm{L}} \cdot \frac{1}{2} \cdot \rho_{1} \cdot \mathbf{u}_{*}^{2} \cdot \frac{\pi}{4} \cdot \mathbf{d}^{2}}{\left(\rho_{\mathrm{s}}-\rho_{\mathrm{l}}\right) \cdot \mathrm{g} \cdot \frac{\pi}{6} \cdot \mathbf{d}^{3} \cdot \psi+\frac{1}{2} \cdot \rho_{\mathrm{s}} \cdot \frac{\pi}{6} \cdot \mathbf{d}^{3} \cdot \psi \cdot \frac{\mathrm{v}_{\mathrm{th}}^{2}}{\mathrm{x}}} \\
& \approx \frac{\mathbf{C}_{\mathbf{L}} \cdot \mathbf{u}_{*}^{2}}{\mathrm{~d} \cdot\left(\mathbf{R}_{\mathrm{sd}} \cdot \mathrm{g}+\frac{1}{2} \cdot \frac{\rho_{\mathrm{s}}}{\rho_{\mathrm{l}}} \cdot \frac{\mathbf{v}_{\mathrm{th}}^{2}}{\mathrm{x}}\right)}
\end{aligned}
$$

Now suppose the distance to stop the particle equals a number of times the thickness of the viscous sub layer, this gives:

$$
L_{R}=\frac{C_{L} \cdot u_{*}^{2}}{d \cdot\left(R_{s d} \cdot g+\frac{1}{2} \cdot \frac{\rho_{s}}{\rho_{l}} \cdot \frac{v_{t h}^{2} \cdot u_{*}}{\alpha \cdot 11.6 \cdot v_{l}}\right)} \cdot\left(1-\frac{C_{v s}}{C_{v b}}\right)
$$

The concentration term is added, because the higher the concentration, the smaller the capacity of the turbulent flow to generate lift forces. When the spatial concentration $\mathrm{C}_{\mathrm{vs}}$ equals the bed concentration $\mathrm{C}_{\mathrm{vb}}$, there will not be any lift forces anymore. For now a linear relation is chosen.

Figure 5 shows this lift ratio at the intersection line speed between the heterogeneous and homogeneous flow regimes for $\alpha=10, C_{v s}=0.175$ and $C_{v b}=0.55$ (which is also used in the graphs), where the intersection line speed can be computed with:

$$
\frac{v_{t} \cdot\left(1-\frac{C_{v s}}{0.175 \cdot(1+\beta)}\right)^{\beta}}{v_{l s}}+8.5^{2} \cdot\left(\frac{1}{\lambda_{1}}\right) \cdot\left(\frac{v_{t}}{\sqrt{g \cdot d}}\right)^{10 / 3} \cdot\left(\frac{\left(v_{1} \cdot g\right)}{v_{l s}}\right)^{1 / 3}=\frac{\lambda_{1} \cdot v_{l s}^{2}}{2 \cdot g \cdot D_{p}}
$$

In reality the intersection line speed will be a bit higher, due to the reduced ELM because of the particle poor viscous sub layer (reduction ELM 50\%-60\%). From Figure 5 it is shown that for very small particles the submerged weight dominates and the lift ratio hardly reaches a value of 1 . For medium sized particles the near wall lift dominates giving a lift ratio above 1 . It must be mentioned that for small diameter pipes the lift ratio never reaches 1 , while the lift ratio increases with increasing pipe diameter. For large particles both submerged weight and kinetic energy dominate, resulting in a lift ratio never reaching 1 . So the conclusion can be drawn that very small and very large particles do not show a collapse of the heterogeneous $E_{\text {rhg }}$, but medium sized particles do, where the collapse is stronger in larger diameter pipes.

So how to model this collapse? The kinetic energy contribution in the heterogeneous flow regime equation is subject to the influence of the near wall lift force, so only this term is reduced. Based on the experiments considered, the reduction is not linear with the lift ratio, but quadratic, resulting in:

$$
E_{r h g}=\frac{v_{t} \cdot\left(1-\frac{C_{v s}}{0.175 \cdot(1+\beta)}\right)^{\beta}}{v_{l s}}+8.5^{2} \cdot\left(\frac{1}{\lambda_{1}}\right) \cdot\left(\frac{v_{t}}{\sqrt{g \cdot d}}\right)^{10 / 3} \cdot\left(\frac{\left(v_{l} \cdot g\right)^{1 / 3}}{v_{l s}}\right)^{2} \cdot\left(1-L_{R}^{2}\right)
$$


When the lift ratio has a value close to 1 , theoretically there are no more collisions with the wall. However not all particles will have exactly the same kinetic energy, so even when the lift ratio is larger than 1, still some particles will have collisions. Therefore a smoothing function is proposed for lift ratio's larger than $70 \%(\zeta=0.5)$, giving:

$$
E_{\text {rhg }}=\frac{v_{t} \cdot\left(1-\frac{C_{v s}}{0.175 \cdot(1+\beta)}\right)^{\beta}}{v_{\text {Is }}}
$$

$$
+8.5^{2} \cdot\left(\frac{1}{\lambda_{1}}\right) \cdot\left(\frac{v_{t}}{\sqrt{g \cdot d}}\right)^{10 / 3} \cdot\left(\frac{\left(v_{1} \cdot g\right)^{1 / 3}}{v_{1 s}}\right)^{2} \cdot(1-\zeta) \cdot \frac{\zeta}{L_{R}^{2}}
$$

Figure 3 and Figure 4 show the decrease of the heterogeneous relative excess hydraulic gradient (heterogeneous flow with near wall lift), based on the lift ratio reduction. It is clear that a larger pipe diameter results in a larger decrease. The reason the $\mathrm{E}_{\mathrm{rhg}}$ does not go to zero, is that the potential energy losses are not affected by the lift ratio.

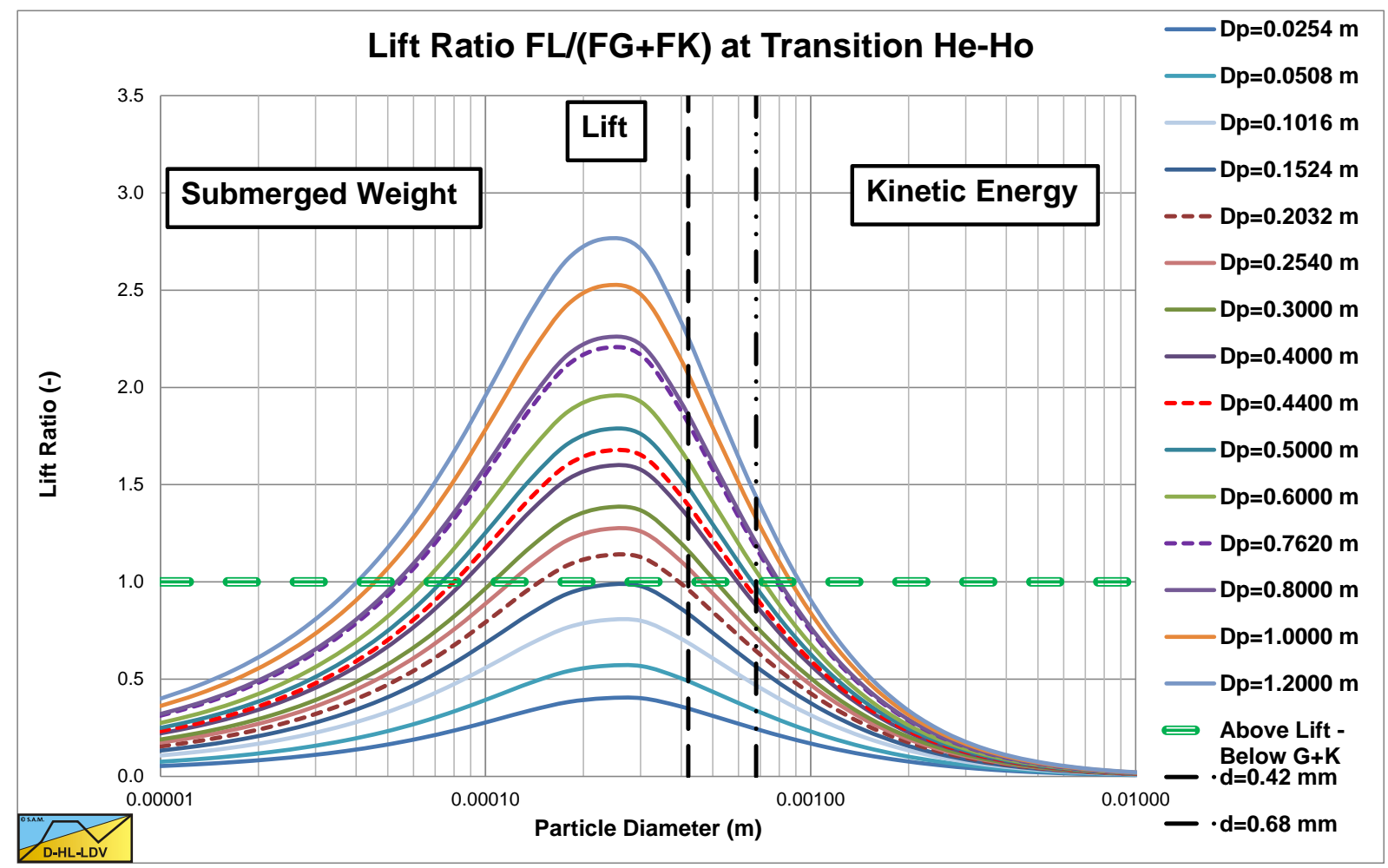

Figure 5: The lift ratio at the transition line speed heterogeneous-homogeneous.

\section{LIMIT DEPOSIT VELOCITY \& CONCENTRATION DISTRIBUTION}

Now that the collapse of the heterogeneous $\mathrm{E}_{\text {rhg }}$ has been modelled, the question is, how does the homogeneous $\mathrm{E}_{\text {rhg }}$ behave? The background of the ELM is that small particles rotate with turbulent eddies. This implies that the kinetic rotational energy of an eddy increases if the mass of the eddy increases. Since the mass of an eddy increases with the mixture density, the rotational energy also increases with the mixture density. So at high line speeds, where turbulence has fully developed, head losses will be proportional with the mixture density. Because of the particle poor viscous sub layer there is a lubrication effect, resulting in a smaller increase, as has been described with equation (7). This equation however assumes that turbulence has fully developed and particles follow the eddies. This may be true at high line speeds, but certainly not at low line speeds. It is also the question whether larger particles will follow this principle. At lower line speeds, the RELM effect will not be fully mobilized. The question is, how much of the RELM effect is mobilized as a function of the line speed? 
To answer this question, first the Limit Deposit Velocity (LDV) is considered. The Limit Deposit Velocity is defined as the line speed above which there is no stationary or sliding bed. The definition of Wilson et al. (1992) is the line speed where a bed starts sliding, the Limit of Stationary Deposit Velocity (LSDV). The latter will only occur if the particle diameter to pipe diameter ratio exceeds a certain value. For small particles, the bed will never start sliding, because it already dissolves into heterogeneous transport, before the shear stress on the bed is large enough to initiate a sliding bed. The two different definitions also give different results. The LDV found by Durand \& Condolios (1952) gives higher values than the LSDV found by Wilson et al. (1992). Goedde (1978) investigated the LDV by measuring the bed height at different line speeds below the LDV and found the LDV by drawing a straight line through the data points in order to obtain the line speed where the bed height is zero. He carried out experiments on plastic, coal, sand and iron ore. He concluded that the LDV found matched the findings of Durand \& Condolios (1952) very well. Durand \& Condolios (1952) in their publications only mention that their LDV is the line speed above which there is no deposit, but with the findings of Goedde (1978) this definition can be made more explicit, no deposit means, nor a stationary deposit, nor a sliding bed.

Miedema \& Ramsdell (2015A) developed a model for the LDV based on the definition of Goedde (1978) and Durand \& Condolios (1952). This definition implies that at the LDV, the spatial concentration at the pipe bottom equals the bed concentration. The advection diffusion equation when in equilibrium shows the balance between the upwards flow of particles due to diffusion and the downwards flow of particles due to gravity (the terminal settling velocity). Wasp et al. (1977) and Doron et al. (1987) use the solution of the advection diffusion equation for low concentrations, while Karabelas (1977) and Kaushal \& Tomita (2002) use the Hunt (1954) approach with upwards liquid flow. Hindered settling is not yet included in the basic solutions, but added by replacing the terminal settling velocity $\mathrm{v}_{\mathrm{t}}$ by the hindered terminal settling velocity $\mathrm{v}_{\mathrm{th}}$. For the diffusivity and the relation between the sediment diffusivity and the turbulent eddy momentum diffusivity different approaches are possible. Using the Lane \& Kalinske (1941) approach, the following equation can be derived for pipe flow:

$$
C_{v S}(r)=C_{v B} \cdot e^{-12 \cdot \frac{v_{t h}}{\beta_{s m} \cdot k \cdot u_{*}} \cdot \frac{r}{D_{p}}}
$$

Now based on the assumption that the diffusivity has to have a value such that at the LDV the concentration at the bottom of the pipe equals the bed concentration (the definition of the LDV), the following equation is derived by Miedema \& Ramsdell (2015B):

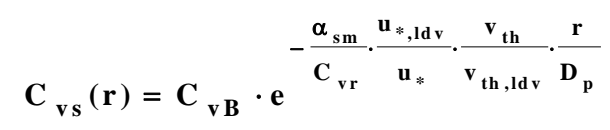

$$
\text { W ith: } \quad \alpha_{\mathrm{sm}}=1.0046+0.1727 \cdot \mathrm{C}_{\mathrm{vr}}-1.1905 \cdot \mathrm{C}_{\mathrm{vr}}^{2}
$$

The settling velocity $\mathrm{v}_{\text {th }}$ is the settling velocity of the particle, based on the properties of the liquid, adjusted for the homogeneous fraction, resulting in the vehicle liquid according to Wasp et al. (1977). The correction factor $\alpha_{\mathrm{sm}}$ appears to depend only on the relative concentration $\mathrm{C}_{\mathrm{vr}}$. The bottom concentration $\mathrm{C}_{\mathrm{vB}}$ is now for line speeds above the LDV:

$$
\mathbf{C}_{\mathbf{v B}}=\mathbf{C}_{\mathbf{v b}} \cdot \frac{\mathbf{u}_{*, \mathrm{Idv}}}{\mathbf{u}_{*}} \cdot \frac{\mathbf{v}_{\text {th }}}{\mathbf{v}_{\text {th }, \mathrm{Idv}}}
$$

Figure 6 shows the concentration profiles for different relative concentrations, adjusted for the circular shape of the pipe at the LDV, compared with data from Kaushal et al. (2005) for a $0.44 \mathrm{~mm}$ particle, giving a reasonable match. Since hindered settling is applied for the average vehicle concentration, applying the local concentration may alter the profiles slightly. Especially at higher concentrations, the hindered settling in the upper half of the pipe will be less than in the bottom half of the pipe, resulting in lower concentrations in the upper half of the pipe and higher concentrations in the lower half of the pipe, as shown in Figure 6. 


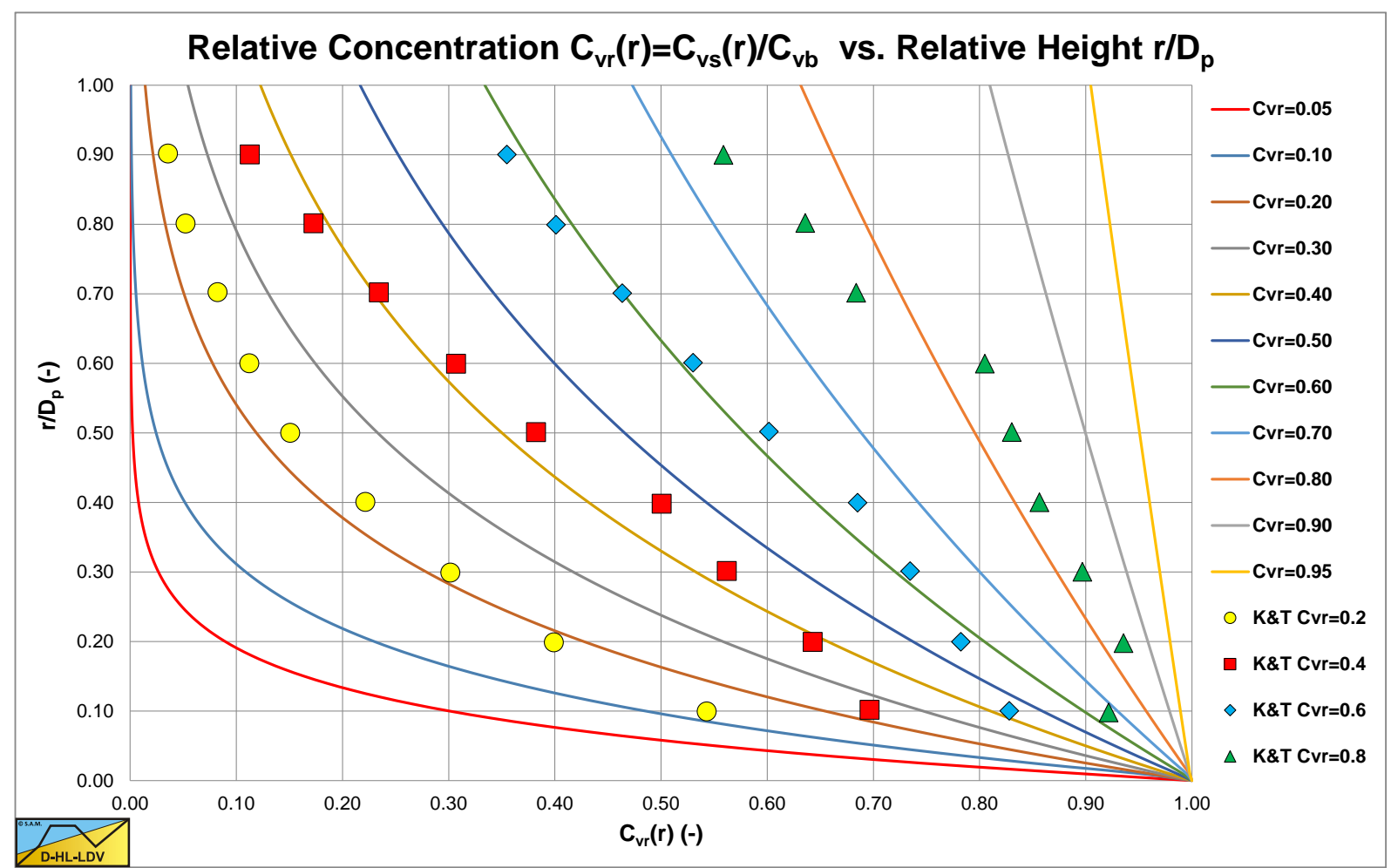

Figure 6: The concentration distribution at the $\mathrm{LDV}$ as a function of the relative concentration.

Now how does this contribute to the mobilization of the RELM? Assume this mobilization m depends on the ratio of the concentration at two levels $r_{1}$ and $r_{2}$ in the pipe, this gives:

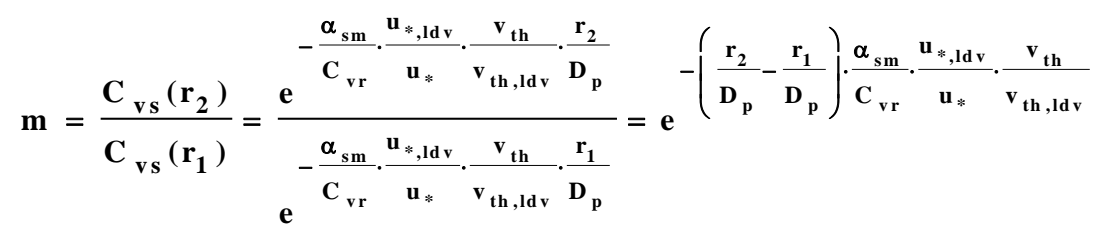

Wasp et al. (1977) use $r_{1}=0.5 \cdot D_{p}$ and $r_{2}=0.92 \cdot D_{p}$ to determine the vehicle fraction, which is not the same as the mobilization factor of the RELM. Different values are tested and the best outcome was found choosing $r_{1}=0.45 \cdot D_{p}$ and $\mathrm{r}_{2}=0.55 \cdot \mathrm{D}_{\mathrm{p}}$, giving:

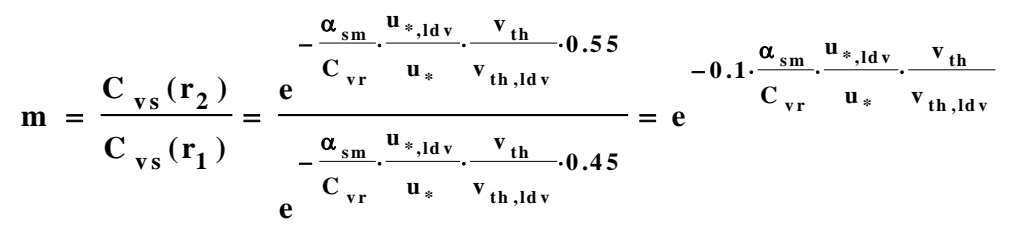

Basically this shows the concentration gradient at the center of the pipe. Figure 3 and Figure 4 show the mobilized homogeneous flow $\mathrm{E}_{\text {rhg }}$ according to:

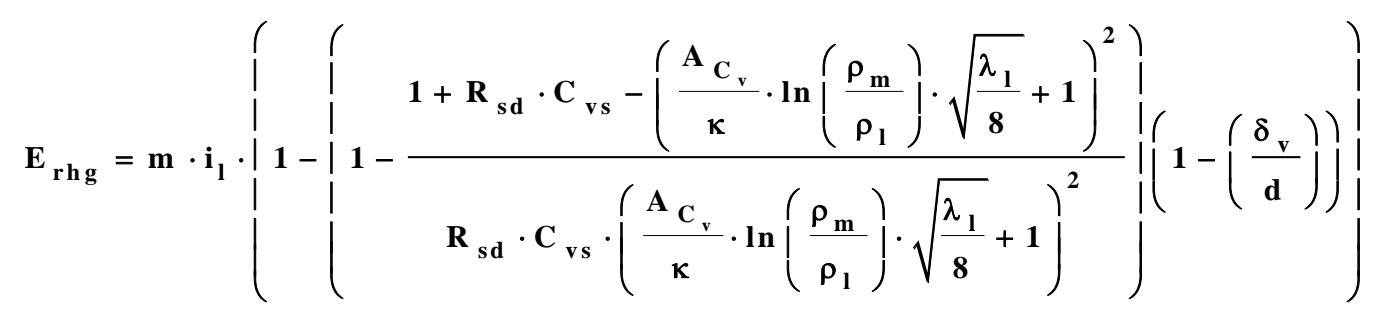




\section{RESULTING RELATIVE EXCESS HYDRAULIC GRADIENT CURVES}

Figure 7 gives an example of the resulting relative excess hydraulic gradient curves for a $0.2032 \mathrm{~m}$ diameter pipe and particles ranging from $0.1 \mathrm{~mm}$ to $10 \mathrm{~mm}$, showing the different flow regimes for the transport (delivered) concentration case.

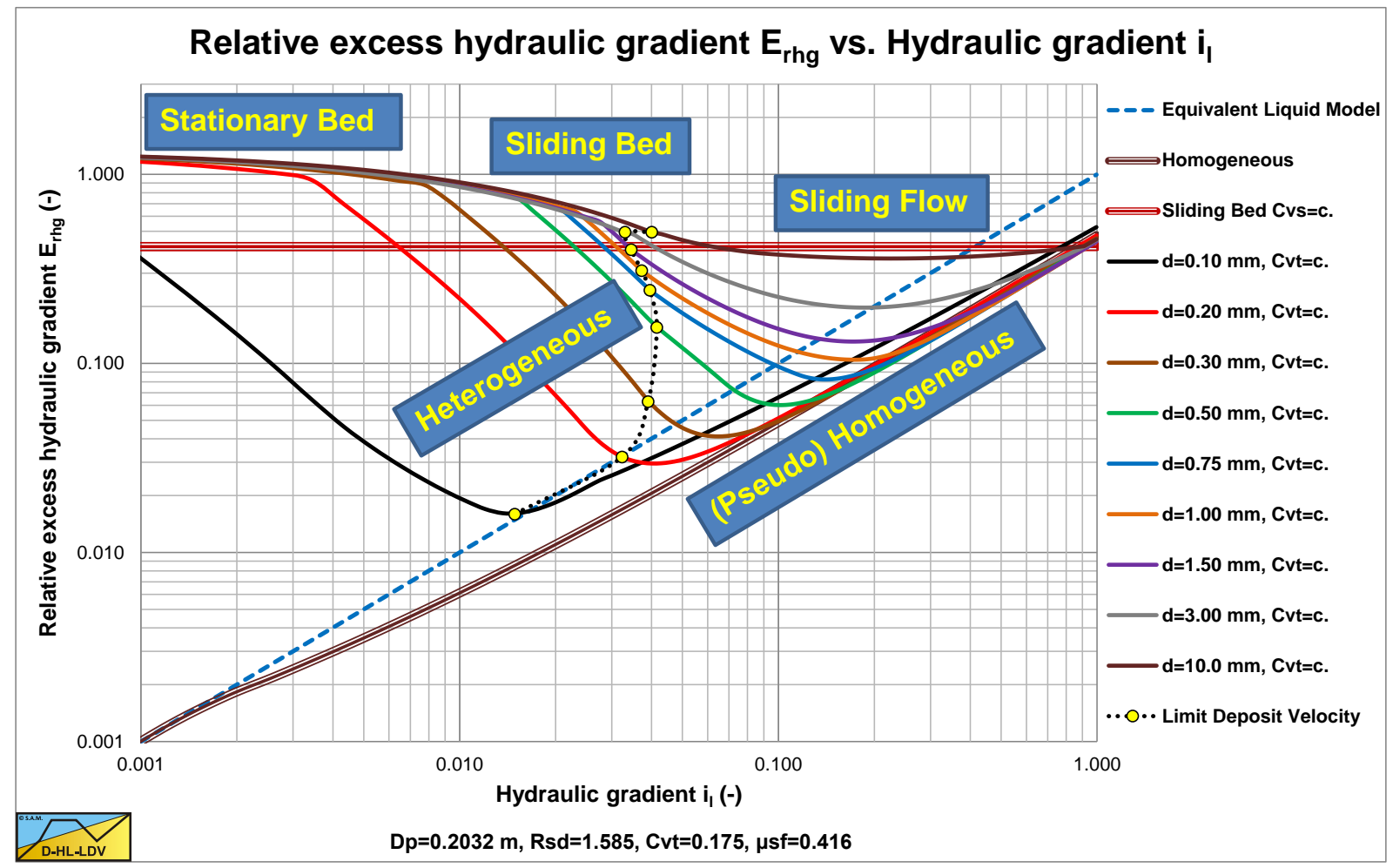

Figure 7: The main flow regimes for constant delivered/transport concentration, pipe diameter $D_{p}=0.2032 \mathrm{~m}$.

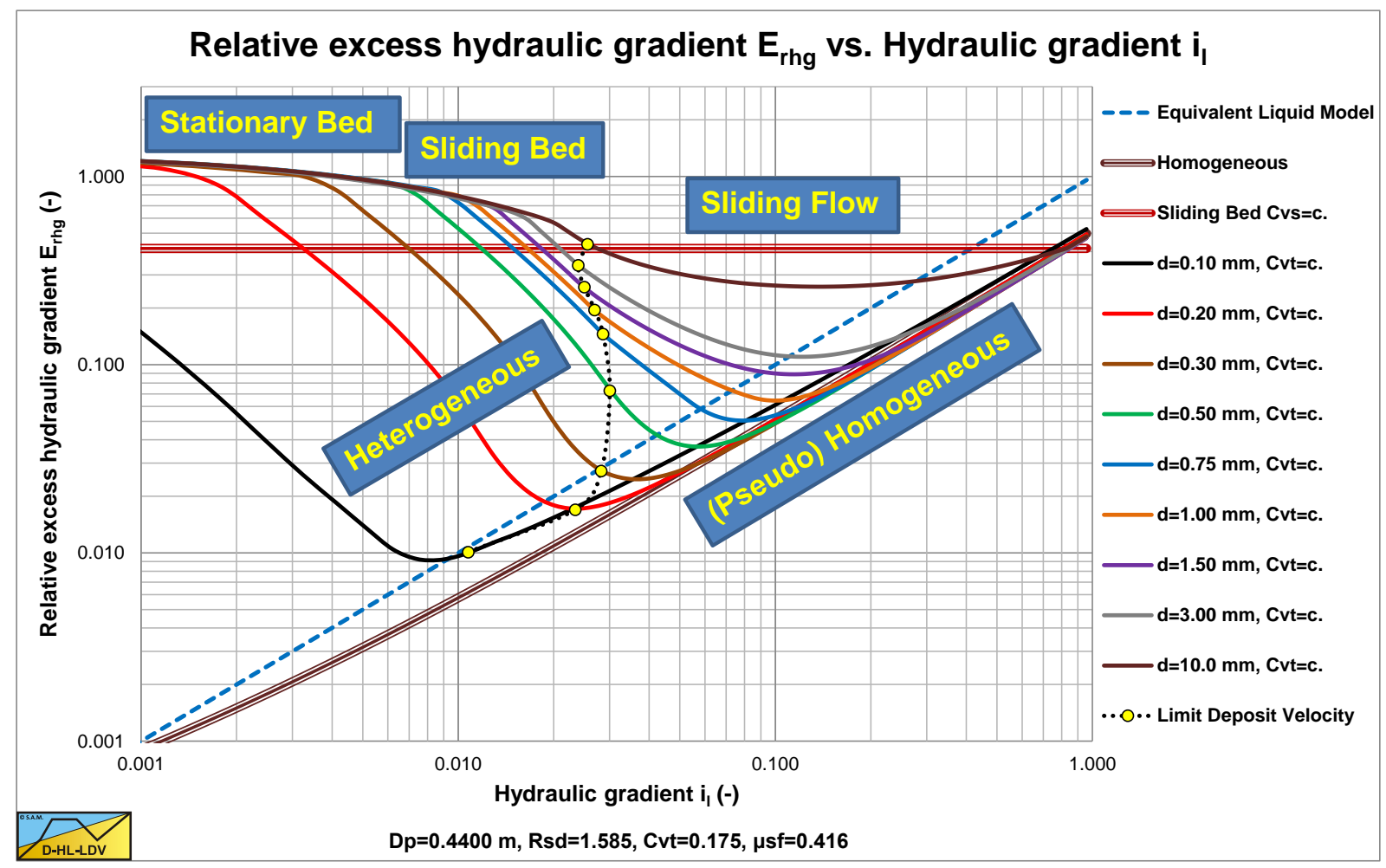

Figure 8: The main flow regimes for constant delivered/transport concentration, pipe diameter $D_{p}=\mathbf{0 . 4 4} \mathrm{m}$. 
Figure 8 shows the resulting relative excess hydraulic gradient curves for a $0.44 \mathrm{~m}$ diameter pipe for the transport (delivered) concentration case. From these two figures it is clear that the larger pipe diameter shows a steeper decrease of the $E_{\mathrm{rhg}}$ in the heterogeneous regime for particles of $0.2 \mathrm{~mm}, 0.3 \mathrm{~mm}$ and $0.5 \mathrm{~mm}$, so medium sands. Very small particles are dominated by the submerged weight, while larger particles are dominated by their kinetic energy.

\section{CONCLUSIONS \& DISCUSSION}

The transition of different flow regimes is important, but the transition of the heterogeneous flow regime to the homogeneous flow regime is very important, due to the fact that this coincides often with operational line speeds. To model this transition it is necessary to have appropriate models for the heterogeneous and the homogeneous flow regimes. The homogeneous flow regime is modelled as a reduced equivalent liquid model (RELM) with mobilization of the reduction based on the ratio between the thicknesses of the viscous sub layer to the particle diameter. For very small particles there is no reduction at low line speeds. The reduction is in effect at higher line speeds. Medium and large particles encounter this reduction at lower line speeds. The heterogeneous flow regime is modelled based on potential and kinetic energy losses, where the potential energy losses are inversely proportional to the line speed and the kinetic energy losses (based on collisions) are inversely proportional to the line speed squared.

At the transition (intersection line speed) of the two flow regimes, the heterogeneous head losses collapse in larger pipe diameters for medium sized particles. It appears that these particles encounter the highest lift forces, compared to their submerged weight and kinetic energy. For very small particles the submerged weight dominates, due to the fact that transition line speed is very small and there is hardly any lift. For very large particles the kinetic energy dominates and the lift is not capable to decelerate the particles. But for medium sized particles, the lift is stronger than the combined effect of submerged weight and kinetic energy. In a $0.2032 \mathrm{~m}$ diameter pipe the collapse of the collisions is still weak, but in a $0.44 \mathrm{~m}$ diameter pipe this is already strong. Larger pipe diameter will most probably show a stronger effect, explaining also why dredging companies state that medium sized particles in large diameter pipes have head losses close to the head losses of pure water.

The RELM is not yet fully mobilized at low line speeds, due to the fact that turbulent eddies are not yet capable of integrating particles in the rotation of the eddies. The higher the line speed, the more the particles become an integrated part of the turbulence (if they are not too large). Based on the definition of the LDV and the concentration distribution equation from the advection diffusion equation, a mobilization factor has been defined for the mobilization of the RELM. The concentration distribution is such that at the LDV the concentration at the bottom of the pipe equals the bed concentration.

It can be stated that the new model explains for the homogeneous behavior of very small particles, regarding the mobilization of the lubrication effect of the particle poor viscous sub layer. It can also be stated that an explanation is found for the collapse of the heterogeneous head losses of medium sized particles in larger pipes, based on the near wall lift and the mobilization of the RELM.

\section{NOMENCLATURE}

\begin{tabular}{|c|c|c|}
\hline $\mathbf{A}_{\mathbf{c v}}$ & Coefficient RELM (default 3) & - \\
\hline $\mathbf{C}_{\mathbf{D}}$ & Particle drag coefficient & - \\
\hline $\mathbf{C}_{\mathbf{L}}$ & Lift coefficient & - \\
\hline $\mathbf{C}_{\mathrm{vt}}$ & Delivered (transport) volumetric concentration & - \\
\hline $\mathbf{C}_{\mathrm{vs}}$ & Spatial volumetric concentration & - \\
\hline $\mathbf{C}_{\mathrm{vb}}$ & Spatial volumetric concentration bed (1-n) & - \\
\hline $\mathbf{C}_{\mathrm{vB}}$ & Spatial concentration at the bottom of the pipe & - \\
\hline $\mathbf{C}_{\mathrm{vr}}$ & Relative spatial concentration & - \\
\hline d & Particle diameter & m \\
\hline $\mathbf{D}_{\mathbf{p}}$ & Pipe diameter & m \\
\hline ELM & Equivalent liquid model & - \\
\hline $\mathbf{E}_{\mathbf{k}}$ & Kinetic energy loss & Nm \\
\hline Erhg & Relative excess hydraulic gradient & - \\
\hline $\mathbf{F}_{\mathbf{L}}$ & Lift force on particle & $\mathbf{N}$ \\
\hline $\mathbf{F}_{\mathbf{G}}$ & Submerged weight of particle & $\mathbf{N}$ \\
\hline $\mathbf{F}_{\mathbf{K}}$ & Kinetic energy deceleration force & $\mathbf{N}$ \\
\hline $\mathbf{g}$ & Gravitational constant $9.81 \mathrm{~m} / \mathrm{s}^{2}$ & $\mathrm{~m} / \mathrm{s}^{2}$ \\
\hline
\end{tabular}




\begin{tabular}{|c|c|c|}
\hline il & Pure liquid hydraulic gradient & $\mathbf{m} / \mathbf{m}$ \\
\hline $\mathbf{i}_{\mathbf{m}}$ & Mixture hydraulic gradient & $\mathbf{m} / \mathbf{m}$ \\
\hline LDV & Limit Deposit Velocity & $\mathbf{m} / \mathbf{s}$ \\
\hline LSDV & Limit of Stationary Deposit Velocity & $\mathbf{m} / \mathbf{s}$ \\
\hline $\mathbf{L}_{\mathbf{R}}$ & Lift ratio & \\
\hline $\mathbf{m}$ & Mobilized RELM factor & \\
\hline $\mathbf{m}_{\mathbf{p}}$ & Mass particle & kg \\
\hline RELM & Reduced equivalent liquid model & \\
\hline $\mathbf{r}, \mathbf{r}_{1}, \mathbf{r}_{2}$ & Vertical distance in pipe & $\mathbf{m}$ \\
\hline $\mathbf{R}$ & Stratification ratio Wilson & - \\
\hline $\mathbf{R}_{\text {sd }}$ & Relative submerged density & - \\
\hline$S_{\text {hr }}$ & Settling velocity Hindered Relative & \\
\hline Srs & Slip velocity Relative Squared & - \\
\hline $\mathbf{u} *$ & Friction velocity & $\mathbf{m} / \mathbf{s}$ \\
\hline $\mathbf{u}$ *,Idv & Friction velocity at LDV & $\mathbf{m} / \mathbf{s}$ \\
\hline Vls & Line speed & $\mathbf{m} / \mathbf{s}$ \\
\hline $\mathbf{v}_{\mathbf{s l}}$ & Slip velocity & $\mathbf{m} / \mathbf{s}$ \\
\hline $\mathbf{v}_{\mathbf{t}}$ & Terminal settling velocity particle & $\mathbf{m} / \mathbf{s}$ \\
\hline Vth & Hindered terminal settling velocity particle & $\mathbf{m} / \mathbf{s}$ \\
\hline $\mathbf{V}_{\text {th, }, \mathbf{d v}}$ & Hindered terminal settling velocity particle at LDV & $\mathbf{m} / \mathbf{s}$ \\
\hline Vov & Velocity at viscous sub layer thickness & $\mathbf{m} / \mathbf{s}$ \\
\hline $\mathbf{x}$ & Distance to decelerate particle & $\mathbf{m}$ \\
\hline$\alpha$ & Number of times thickness viscous sub layer & - \\
\hline $\boldsymbol{\alpha}_{\mathrm{sm}}$ & Coefficient concentration distribution & - \\
\hline $\boldsymbol{\beta}$ & Richardson \& Zaki hindered settling power & - \\
\hline$\beta_{\mathrm{sm}}$ & Diffusivity factor & - \\
\hline $\boldsymbol{\delta}_{\mathrm{v}}$ & Viscous sub layer thickness & $\mathbf{m}$ \\
\hline$\kappa$ & Von Karman constant (about 0.4 ) & \\
\hline$\kappa_{\mathrm{C}}$ & Concentration eccentricity factor & - \\
\hline$\lambda_{1}$ & Darcy Weisbach friction factor liquid & - \\
\hline$\mu_{\mathrm{sf}}$ & Sliding friction factor & \\
\hline$\rho_{1}$ & Density liquid & $\operatorname{ton} / \mathrm{m}^{3}$ \\
\hline$\rho_{\mathbf{m}}$ & Density mixture & $\operatorname{ton} / \mathbf{m}^{3}$ \\
\hline$\rho_{\mathrm{s}}$ & Density solid & $\operatorname{ton} / \mathrm{m}^{3}$ \\
\hline $\mathbf{v}_{\mathbf{I}}$ & Kinematic viscosity liquid & $\mathbf{m}^{2} / \mathrm{s}$ \\
\hline$\Psi$ & Shape factor & - \\
\hline$\zeta$ & Smoothing parameter lift ratio & - \\
\hline $\boldsymbol{\theta}$ & Dimensionless lift ratio coefficient & \\
\hline
\end{tabular}

\section{BIBLIOGRAPHY}

Blythe, C., \& Czarnotta, Z. (1995). Determination of hydraulic gradient for sand slurries. 8th International Freight Pipeline Society Symposium, (pp. 125-130). Pittsburg, USA.

Clift, R., Wilson, K. C., Addie, G. R., \& Carstens, M. R. (1982). A mechanistically based method for scaling pipeline tests for settling slurries. Hydrotransport 8 (pp. 91-101). Cranfield, UK.: BHRA Fluid Engineering.

Doron, P., Granica, D., \& Barnea, D. (1987). Slurry flow in horizontal pipes, experimental and modeling. International Journal of Multiphase Flow, Vol. 13, No. 4., 535-547.

Durand, R., \& Condolios, E. (1952). Etude experimentale du refoulement des materieaux en conduites en particulier des produits de dragage et des schlamms. Deuxiemes Journees de l'Hydraulique., 27-55.

Fuhrboter, A. (1961). Über die Förderung von Sand-Wasser-Gemischen in Rohrleitungen. Mitteilungen des Franzius-Instituts, H. 19.

Goedde, E. (1978). To the critical velocity of heterogeneous hydraulic transport. Hydrotransport 5 (pp. B4-81-B498). Cranfield, Bedford, England: BHRA.

Hunt, J. N. (1954). The turbulent transport of suspended sediment in open channels. Royal Society of London, Proc. Series A, Vol. 224(1158)., 322-335.

Karabelas, A. J. (1977). Vertical Distribution of Dilute Suspensins in Turbulent Pipe Flow. AIChE Journal, Vol. 23(4)., 426-434. 
Kaushal, D. R., \& Tomita, Y. (2002). Solids concentration profiles and pressure drop in pipeline flow of multisized particulate slurries. International Journal of Multiphase Flow, Vol. 28., 1697-1717.

Kaushal, D. R., Sato, K., Toyota, T., Funatsu, K., \& Tomita, Y. (2005). Effect of particle size distribution on pressure drop and concentration profile in pipeline flow of highly concentrated slurry. International Journal of Multiphase Flow, Vol. 31., 809-823.

Lane, E. W., \& Kalinske, A. A. (1941). Engineering calculations of suspended sediment. Trans. Am. Geophysics Union, Vol. 20(3)., 603-607.

Miedema, S. A. (2014A). An analytical approach to explain the Fuhrboter equation. Maritime Engineering, 17.

Miedema, S. A. (2014B). An analysis of slurry transport at low line speeds. ASME 2014 33rd International Conference on Ocean, Offshore and Arctic Engineering, OMAE. (p. 11). San Francisco, USA.: ASME.

Miedema, S. A. (2015A). A head loss model for homogeneous slurry transport for medium sized particles. Journal of Hydrology \& Hydrodynamics, Vol. 63(1)., 1-12.

Miedema, S. A. (2015B). Head Loss Model for Slurry Transport in the Heterogeneous Regime. Journal of Ocean Engineering. Accepted.

Miedema, S. A. (2015C). THE SLIP RATIO OR HOLDUP FUNCTION IN SLURRY TRANSPORT. Dredging Summit and Expo 2015. (p. 12). Houston, Texas, USA.: WEDA.

Miedema, S. A., \& Matousek, V. (2014). An explicit formulation of bed friction factor for sheet flow. International Freight Pipeline Society Symposium, 15th. (p. 17). Prague, Czech Republic: IFPS.

Miedema, S. A., \& Ramsdell, R. C. (2013). A Head Loss Model for Slurry Transport based on Energy Considerations. World Dredging Conference XX (p. 14). Brussels, Belgium: WODA.

Miedema, S. A., \& Ramsdell, R. C. (2014A). An Analysis of the Hydrostatic Approach of Wilson for the Friction of a Sliding Bed. WEDA/TAMU (p. 21). Toronto, Canada: WEDA.

Miedema, S. A., \& Ramsdell, R. C. (2014B). The Delft Head Loss \& Limit Deposit Velocity Model. Hydrotransport (p. 15). Denver, USA.: BHR Group.

Miedema, S. A., \& Ramsdell, R. C. (2015A). The Limit Deposit Velocity Model, a New Approach. Journal of Hydrology \& Hydromechanics, Vol 63(4), 273-286.

Miedema, S. A., \& Ramsdell, R. C. (2015B). The Delft Head Loss \& Limit Deposit Velocity Framework. Journal of Dredging Engineering, Vol. 15(20., 28.

Ramsdell, R. C., \& Miedema, S. A. (2013). An overview of flow regimes describing slurry transport. WODCON $X X$ (p. 15). Brussels, Belgium.: WODA.

Schlichting, H. (1968). Boundary layer theory. 6th ed. New York: McGraw-Hill.

Shook, C., \& Roco, M. (1991). Slurry Flow, Principles \& Practice. Boston: Butterworth Heineman.

Talmon, A. (2011). Hydraulic Resistance of Sand-Water Mixture Flow in Vertical Pipes. T\&S, Transport and Sedimentation of Solid Particles (pp. 137-147). Wroclaw, Poland: T\&S.

Talmon, A. (2013). Analytical model for pipe wall friction of pseudo homogeneous sand slurries. Particulate Science \& technology: An International Journal, 264-270.

Thomas, A. (1976). SCALE-UP METHODS FOR PIPELINE TRANSPORT OF SLURRIES. International Journal of Mineral Processing, Vol. 3., 51-69.

Thomas, D. G. (1962). Transport Characteristics of Suspensions: Part VI. Minimum velocity for large particle size suspensions in round horizontal pipes. A.I.Ch.E. Journal, Vol.8(3)., 373-378.

Wasp, E. J. (1963). Cross country coal pipeline hydraulics. Pipeline News., 20-28.

Wasp, E. J., Kenny, J. P., \& Gandhi, R. L. (1977). Solid liquid flow slurry pipeline transportation. Transactions Technical Publications.

Whitlock, L., Wilson, K. C., \& Sellgren, A. (2004). Effect of near-wall lift on frictional characteristics of sand slurries. Hydrotransport 16 (pp. 443-454). Cranfield, UK.: BHR Group.

Wilson, K. C., \& Sellgren, A. (2003). Interaction of Particles and Near-Wall Lift in Slurry Pipelines. Journal of Hydraulic Engineering, Vol. 129., 73-76.

Wilson, K. C., \& Sellgren, A. (2010). Behavior of intermediate particle slurries in pipelines. Hydrotransport 18 (pp. 117-128). Rio de Janeiro: BHR Group.

Wilson, K. C., Addie, G. R., \& Clift, R. (1992). Slurry Transport using Centrifugal Pumps. New York: Elsevier Applied Sciences.

Wilson, K. C., Addie, G. R., Sellgren, A., \& Clift, R. (2006). Slurry transport using centrifugal pumps. New York: Springer Science+Business Media Inc. 